\title{
Prevalence of COVID-19 in medical school and residency in Porto Alegre, RS
}

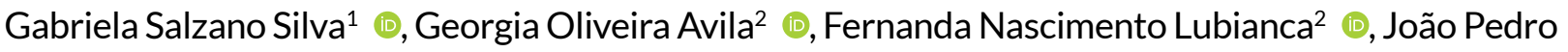

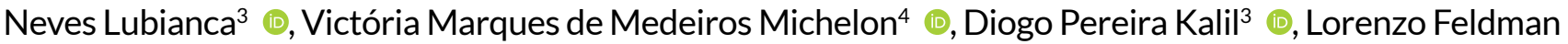

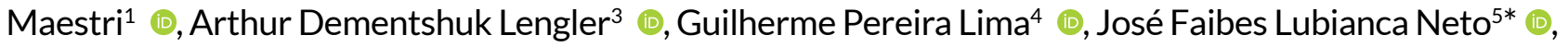 \\ Brazilian Multicenter University Study Group on Prevention and Combating COVID-19
}

\begin{abstract}
SUMMARY
OBJECTIVE: A multicentric, cross-sectional study was carried out to determine the prevalence and risk factors for Coronavirus disease 2019 in medical students and residents from four universities and affiliated hospitals in Brazil.

METHODS: A survey about contamination risk and symptoms was sent to all participants through email and WhatsApp. Prevalence was measured by the self-report of positive polymerase chain reaction or serological test. Univariate and multivariate analyses were performed, and odds ratio and 95\% confidence interval were calculated.

RESULTS: Prevalence of infection by Sars-CoV-2 was 14.9\% (151/1011). The disease was more prevalent in residents and interns than in undergraduate students. Contact with an infected relative outside the hospital or with colleagues without using personal protective equipment was associated with higher contamination. Contact with patients without wearing goggles and higher weekly frequency of contact were the two factors independently associated with the infection by Coronavirus disease 2019 in the multivariate analysis.

CONCLUSIONS: Medical students, interns, and residents have a higher prevalence of Coronavirus disease 2019 than the general population, in which the last two groups are significantly at higher risk. Contacting patients at a higher weekly frequency increases the risk for infection. The use of goggles should be reinforced when contacting patients.
\end{abstract}

KEYWORDS: Sars-CoV-2. Prevalence. Students, Medical. Medical Residency. Internship.

\section{INTRODUCTION}

The pandemic of the coronavirus disease 2019 (COVID-19) caused by Sars-CoV-2 emerged in China and can clinically vary from a mild cold to a multisystemic disease ${ }^{1}$. The disease is manifested by a variety of symptoms such as fever, dry cough, dyspnea, fatigue, body aches, headaches, anosmia, and ageusia which usually progress to spontaneous remission ${ }^{2}$. According to COVID-19 data from the World Health Organization (WHO), from September 07, 2021, over 221 million cases were confirmed and more than 4 million deaths occurred worldwide, and 12.4 million confirmed cases and over 500,000 of those deaths occurred in Brazil ${ }^{3}$. New hygiene habits were adopted from public policies recommended by WHO, including the use of masks and social distancing ${ }^{4}$. As part of these measures, on-site classes were also suspended and the traditional learning strategies underwent several changes at all levels of education in Brazil including medical courses ${ }^{5,6}$.

Healthcare professionals face a greater risk of infection. A study carried out in a hospital in Wuhan identified an infection rate of $29 \%$ among healthcare professionals ${ }^{7}$. Investigations about deaths of doctors related to the infection due to their work on the front line showed fatalities of professionals from all healthcare areas, especially in men with an average age of 63.5 years $^{8}$. A study from the time of the previous SARS pandemic showed that medical students who did not have

\footnotetext{
${ }^{1}$ Universidade Federal de Ciências da Saúde de Porto Alegre, School of Medicine - Porto Alegre (RS), Brazil.

${ }^{2}$ Pontifícia Universidade Católica do Rio Grande do Sul, School of Medicine - Porto Alegre (RS), Brazil.

${ }^{3}$ Universidade Federal do Rio Grande do Sul, School of Medicine - Porto Alegre (RS), Brazil.

${ }^{4}$ Universidade Luterana do Brasil, School of Medicine - Porto Alegre (RS), Brazil.

${ }^{5}$ Universidade Federal de Ciências da Saúde de Porto Alegre, Graduate Program in Pediatrics at UFCSPA, Chief of Othorinolaryngology Service at Hospital Santa Casa de Misericórdia - Porto Alegre (RS), Brazil.

*Corresponding author: jlubianca@ufcspa.edu.br; lubianca@otorrinospoa.com.br Conflicts of interest: the authors declare there is no conflicts of interest. Funding: none.

Received on November 11, 2021. Accepted on November 17, 2021.
} 
contact with patients did not contract SARS'. The purpose of this study was to determine the prevalence of COVID-19 among medical students in different stages of their medical education. The prevalence of COVID-19 in this group of people was evaluated in regard to the use of personal protective equipment (PPE), as well as to the frequency of exposure and to the point-of-care of patients in general and of patients knowingly contaminated.

\section{METHODS}

This is a multicentric, cross-sectional study carried out in four medical schools located in the Porto Alegre (Brazil) and their respective teaching hospitals from August to December 2020. Both positive polymerase chain reaction (PCR) and serological tests were considered COVID-19 infection. The prevalence of the virus was compared in three groups: undergraduate students (1st to end of 4th year of study), interns (5th and 6th year of study), and residents from four Medical Schools. Additionally, the prevalence of COVID-19 was evaluated according to the use of PPE (isolation gowns, N95 masks, caps, goggles, and face shields), the frequency of exposure (months of the year and weeks in the last month), and the point-ofcare (outpatient facility, emergency care settings, intensive care unit [ICU]) of patients in general as well as of patients known to be infected (a positive PCR test). The selection of the sample included all the undergraduate students and interns enrolled in these universities and the residents from the respective affiliated hospitals contacted through email and WhatsApp. All students, interns, and residents who completed the questionnaire within the allotted time for the data collection were included in the study. Data were tabulated and double-checked. Variables were analyzed in a descriptive way. Descriptive analysis was performed for quantitative variables (averages, standard deviation, and medians). For the qualitative variables, absolute and relative frequencies were calculated. The chi-squared test and the Fisher's exact test were applied to test homogeneity among the proportions. The univariate and multivariate logistic regression model was employed to assess COVID-19 predictors and the odds ratio, using SPSS, version 17.0 (Chicago, IL, USA). The significance level used for the tests was $5 \%$. All participants signed an informed consent, and data were kept confidential. The research was approved by the ethics committee/institutional review board of the coordinating center and the other participant universities (CAAE number 36498920.3.1001.5345). The individual projects were forwarded with the endorsement of the clinical management from all four participant hospitals.

\section{RESULTS}

The participants who were considered "COVID-19-positive" were the ones who had either a positive PCR test or a positive serology test for COVID-19, totalizing 151 cases. The remaining group ( $\mathrm{N}=860)$ was considered the "COVID-19-negative" group.

Concerning the course internships, a greater incidence of COVID-19 was found in residents when compared with other students $(\mathrm{p}<0.001)$. There was no difference in frequencies comparing residents and interns and also among different universities (Table 1).

The only symptom that showed significant difference between the two groups was dysgeusia or anosmia, which displayed higher incidence among the COVID-19-positive group, emphasizing their high specificity for COVID-19.

There was a greater prevalence of the virus by contact with a relative (father/mother/siblings/uncle/brother-in-law) or a patient $(\mathrm{p}=0.002)$ when compared with other types of contact (spouse/partner, colleague, classmates, or other). In regard to the practical activities with patients and to the workplace, the highest frequency of COVID-19 was detected in participants who were caring for patients $(\mathrm{p}=0.001)$. Moreover, the type of care associated with the highest risk of being infected took place in emergency care settings $(\mathrm{p}=0.007)$.

With respect to the exposure time frame, a higher incidence of infection in those participants with 3-month of contact and also in those with 6-month of contact was found. There was a minimal difference found concerning the number of shifts worked or the number of days worked. In terms of regularity of the contact, there was a higher incidence of participants with COVID-19 for those who had contact with patients from 4 to 6 times a week when compared with the group that did not have the virus.

The participants' contact with patients known to be infected by the SARS-Cov-2 was divided in terms of regularity: monthly (since March 2020) and weekly (in the last month). There was

Table 1. Absolute and relative frequency of the university and apprentice stage in relation to the COVID-19 group.

\begin{tabular}{l|c|c|c|c|c}
\multirow{2}{*}{ Variable } & \multicolumn{4}{|c|}{ COVID-19 } & \multirow{2}{*}{} \\
\cline { 2 - 5 } & \multicolumn{2}{|c|}{ Negative (n=860) } & \multicolumn{2}{c}{ Positive (n=151) } & \multirow{2}{*}{$\mathbf{p}^{*}$} \\
\cline { 2 - 5 } & $\mathbf{n}$ & $\%$ & $\mathbf{n}$ & $\%$ & \\
\cline { 1 - 5 } $\begin{array}{l}\text { Apprentice } \\
\text { stage }\end{array}$ & & & & & \multirow{2}{*}{$<0.001$} \\
\hline Undergraduate & 502 & 58.4 & 62 & 41.1 & \\
\hline Intern & 202 & 23.5 & 34 & 22.5 & \\
\hline Resident & 156 & 18.1 & 55 & 36.4 & \\
\hline
\end{tabular}

(*) Descriptive level of probability of the chi-squared test. 
a statistical difference when the frequency of the contact was from 4 to 6 times a week per month year-round $(\mathrm{p}<0.001)$ or from 4 to 6 days a week in the last month $(\mathrm{p}<0.001$; see Table 2$)$.

Concerning the use of PPE, there was a lower incidence of infection with the use of goggles in outpatient facilities and greater incidence while using gloves and caps in hospital settings having COVID-19-positive group when compared with the COVID-19-negative group. There was a higher frequency of COVID-19 in respondents who did not use PPE when they were in contact with colleagues inside the workplace $(\mathrm{p}<0.001)$.

In Table 3, it was observed that subjects from 26 to 29 years of age and those over 30 years of age displayed 1.92 and 3.26 greater probability of contracting COVID-19, respectively, than the students between 18 and 22 years of age. The residents showed a probability 2.10 times greater than the undergraduate students of having COVID-19. Participants with activities in emergency care settings displayed a probability 1.74 times greater of having been infected by the virus than the ones without this type of activity. Undergraduate students and residents in contact with patients $2-3$ times a week or for more

Table 2. Absolute and relative frequency of the number of practical activities with patients who were knowingly infected, in relation to the COVID-19 group.

\begin{tabular}{|c|c|c|c|c|c|}
\hline \multirow{3}{*}{ Variable } & \multicolumn{4}{|c|}{ COVID-19 } & \multirow{3}{*}{$\mathbf{p}^{*}$} \\
\hline & \multicolumn{2}{|c|}{ Negative } & \multicolumn{2}{|c|}{ Positive } & \\
\hline & $\mathbf{n}$ & $\%$ & n & $\%$ & \\
\hline \multicolumn{5}{|c|}{ Activities with infected patients } & 0.064 \\
\hline Yes & 329 & 59.5 & 81 & 68.6 & \\
\hline No & 224 & 40.5 & 37 & 31.4 & \\
\hline \multicolumn{5}{|c|}{ Weekly regularity of the contact } & $<0.001$ \\
\hline Only once & 52 & 16.0 & 6 & 7.5 & \\
\hline Total of 2-5 times & 133 & 40.8 & 18 & 22.5 & \\
\hline Once a week & 40 & 12.3 & 8 & 10.0 & \\
\hline 2-3 days a week & 51 & 15.6 & 16 & 20.0 & \\
\hline 4-6 days a week & 34 & 10.4 & 25 & 31.3 & \\
\hline Every day of the week & 16 & 4.9 & 7 & 8.8 & \\
\hline \multicolumn{5}{|c|}{ Monthly regularity of the contact } & $<0.001$ \\
\hline No contact & 68 & 21.3 & 13 & 16.1 & \\
\hline Only once & 76 & 23.8 & 11 & 13.6 & \\
\hline Total of $2-5$ times & 72 & 22.6 & 13 & 16.1 & \\
\hline Once a week & 36 & 11.3 & 12 & 14.8 & \\
\hline 2-3 days a week & 40 & 12.5 & 11 & 13.6 & \\
\hline 4-6 days a week & 20 & 6.3 & 14 & 17.3 & \\
\hline Every day of the week & 7 & 2.2 & 7 & 8.6 & \\
\hline
\end{tabular}

$\left({ }^{*}\right)$ Descriptive level of probability of the chi-squared test. than 4 days a week displayed 2.21 and 4.50 higher probability of contracting COVID-19 than the students whose contact was only once a week. Wearing goggles in outpatient facilities decreased the probability of having COVID- 19 by $64 \%$ while the nonuse increased the chance of having the virus by 2.78 times. The participants who wore gloves and caps in hospitalization settings had, respectively, 2.57 and 3.22 greater probability of having the virus in relation to the ones who did not use these items. Finally, the participants who had contact with colleagues without using any PPE had a probability of contracting COVID-19 at a rate of 3.22 times greater than the ones who wore PPE in such situations.

The variables which presented some significance in the univariate analysis were selected for the study of the multivariate. Only variables measured in both groups (COVID-positive and COVID-negative) were considered. Through the use of a multivariate logistic model with the "stepwise" variable selection, it was demonstrated that the variables "regularity of contact" and "use of goggles in outpatient facilities (protection factor)" were the only ones that presented an independent association with the prevalence of positive test for COVID-19 (Table 3). The respondents with contact higher than 4 days a week had a chance of contracting COVID-19 3.43 times higher than those with contact at least once a week in the last month. The students who did not wear goggles in outpatient facilities displayed a chance of having COVID-19 4.35 times higher than the ones who wore this PPE.

\section{DISCUSSION}

The exposure of healthcare professionals to SARS-COV-2 is known to be greater than in the general population. The hospital work and the direct contact with infected patients are the major elements that contributed to the greater contamination rate observed in this group.

Some evidence suggests that students and younger doctors adopt fewer protective measures both in relation to the use of PPE and to the low adhesion to behavioral methods when they are compared with more experienced doctors ${ }^{10}$. In this study, the highest prevalence of cases with COVID-19 occurred in participants who were over 30 years of age. In contrast, the highest proportion of respondents without COVID-19 was in participants between 18 and 22 years of age. Residents are generally older than undergraduate students and are obviously more exposed due to heavier workload. This result was also expected probably because undergraduate students, generally younger, had no practical activities during a significant part of the pandemic. 
Table 3. Odds ratio value and confidence interval $(\mathrm{Cl})$ to $95 \%$ of the univariate and multivariate models of the selected variables for the prediction of COVID-19.

\begin{tabular}{|c|c|c|c|c|}
\hline \multirow{2}{*}{ Variable } & \multicolumn{2}{|c|}{ Univariate } & \multicolumn{2}{|c|}{ Multivariate } \\
\hline & Odds ratio $(95 \% \mathrm{Cl})$ & $\mathbf{p}$ & Odds ratio $(95 \% \mathrm{Cl})$ & $\mathbf{p}$ \\
\hline \multicolumn{5}{|l|}{ Age } \\
\hline 18-22 years old & 1.00 & - & & \\
\hline 23-25 years old & $1.34(0.85 ; 2.12)$ & 0.260 & & \\
\hline 26-29 years old & $1.92(1.18 ; 3.11)$ & 0.008 & & \\
\hline Over 30 years old & $3.26(1.88 ; 5.65)$ & $<0.001$ & & \\
\hline \multicolumn{5}{|l|}{ Apprentice stage } \\
\hline Undergraduate & 1.00 & - & & \\
\hline Internship & $0.73(0.45 ; 1.15)$ & 0.177 & & \\
\hline Residency & $2.10(1.30 ; 3.37)$ & 0.002 & & \\
\hline \multicolumn{5}{|c|}{ Activity in emergency care settings } \\
\hline No & 1.00 & - & & \\
\hline Yes & $1.74(1.16 ; 2.59)$ & 0.007 & & \\
\hline \multicolumn{5}{|c|}{ Weekly regularity of contact } \\
\hline Up to once a week & 1.00 & - & 1.00 & \\
\hline $2-3$ days & $2.21(1.13 ; 4.32)$ & 0.021 & $1.86(0.87 ; 3.95)$ & 0.109 \\
\hline 4 days or more & $4.50(2.53 ; 8.02)$ & $<0.001$ & $3.43(1.78 ; 6.60)$ & $<0.001$ \\
\hline \multicolumn{5}{|c|}{ Use of goggles in outpatient facilities } \\
\hline No & 1.00 & - & 1.00 & - \\
\hline Yes & $0.36(0.15 ; 0.85)$ & 0.020 & $0.23(0.07 ; 0.78)$ & 0.018 \\
\hline \multicolumn{5}{|c|}{ Use of gloves in hospital settings } \\
\hline No & 1.00 & - & & \\
\hline Yes & $2.57(1.45 ; 4.53)$ & 0.001 & & \\
\hline \multicolumn{5}{|c|}{ Use of caps in hospital settings } \\
\hline No & 1.00 & - & & \\
\hline Yes & $3.22(1.63 ; 6.36)$ & $<0.001$ & & \\
\hline \multicolumn{5}{|c|}{ Contact with colleagues without PPE } \\
\hline No & 1.00 & & & \\
\hline Yes & $3.22(1.52 ; 6.81)$ & & & \\
\hline
\end{tabular}

PPE: personal protective equipment.

Other countries have also evaluated the prevalence of contamination among healthcare professionals. Houlihan et al. evaluated 200 healthcare professionals, with a contraction rate of $44 \%$ and a higher tendency of contamination among participants younger than 30 years of age, which contradicts the results obtained in our study ${ }^{11}$.

An important finding, which determined higher prevalence of COVID-19, is the recognition that the infected index-person was a direct relative or patient being cared for by the student/resident. Consequently, the contamination can occur at home or outside the hospital.
A systematic review carried out to assess the impact of COVID-19 in healthcare workers included 64 separate studies, concluding that not washing hands constantly, using PPE insufficient or inadequately, working longer hours, and working in high-risk areas were the independent risk factors for COVID-19 infection ${ }^{12}$. The present analysis found a greater occurrence of the virus in professionals who did not use PPE and/or in professionals who had contact with colleagues without using PPE. This shows the importance of keeping the protective equipment on at all times in hospital settings. In addition, there was a greater 
incidence of infection in those who worked for 3 and 6 months if compared with the ones without the virus. The frequency of exposure was decisive (weekly and monthly contact) for the contamination, with a greater proportion of cases with infection in those with more contact (from 4 to 6 times per week).

In relation to the symptoms, the most significant finding is the association of the virus with anosmia which, in accordance with the current available literature, suggests that hyposmia/ anosmia is the most predictive symptom for COVID-19. A study carried out in two maternity wards in London evaluated the IgG seropositivity in 200 healthcare professionals (anesthesiologists, obstetricians, and midwives). Seroconversion rate was $14.5 \%$ (29/200 - 95\%CI 9.9-20.1). Among the symptomatic subjects, anosmia was the only symptom predictor of seroconversion $(\mathrm{p}<0.001)^{13}$.

Our study has limitations. Although it is multicentric, it is restricted to the universities in the Porto Alegre area at Rio Grande do Sul. These results cannot be generalized to other universities in our or other states of the country, since the pandemic context can present geographical variations. Moreover, recall bias is a limitation of this study design. The COVID-19 diagnosis was based on the report of the participants and their positive tests (PCR-RT or serology) were not confirmed. These limitations do not invalidate the study, which should be regarded as an instrument to promote new hypotheses to be tested in investigations specifically designed for this purpose.

\section{CONCLUSIONS}

The prevalence of COVID-19 antibodies was higher in the studied sample than in the general population of Porto Alegre, Rio Grande do Sul. The prevalence of COVID-19 was higher among residents and interns than in undergraduate students. Contact with an infected relative outside the hospital was associated with a higher occurrence of contamination. Anosmia was the only symptom more prevalent in the COVID-19-positive group. Contact with patients without wearing goggles and higher weekly frequency of contact were independent factors associated with the infection by COVID-19.

\section{REFERENCES}

1. Wiersinga WJ, Rhodes A, Cheng AC, Peacock SJ, Prescott HC. Pathophysiology, transmission, diagnosis, and treatment of coronavirus disease 2019 (COVID-19): a review. JAMA. 2020;324(8):782-93. https://doi.org/10.1001/jama.2020.12839

2. Coronavirus. [cited 2021 March 28]. In: WHO int [Internet] Available from: https://www.who.int/health-topics/ coronavirus\#tab=tab_3

\section{MEMBER OF BRAZILIAN MULTICENTER UNIVERSITY STUDY GROUP ON PREVENTION AND COMBATING COVID-19}

Maria Eduarda Deon Ceccato: Pontifícia Universidade Católica do Rio Grande do Sul (PUCRS), School of Medicine - 6681 Ipiranga Avenue, Porto Alegre, RS, Brazil; Thomas Maahs: ENT resident, Department of Otolaryngology Head and Neck Surgery, Hospital São Lucas da PUCRS, RS, Brazil; Marina Paese Pasqualini: ENT resident, Department of Otolaryngology, Hospital Santa Casa de Misericórdia, Porto Alegre, RS, Brazil; Renato Roithman: Adjunct Professor of Otolaryngology FAMED/ULBRA/RS; Alexandre Vontobel Padoin: Graduate Program in Medicine and Health Sciences, School of Medicine, PUCRS, Porto Alegre, RS, Brazil; and Jaqueline Neves Lubianca: Associate Professor of Obstetrics and Gynecology - Federal University of Rio Grande do SUL (UFRGS). Graduate Program of Ciências da Saúde: Ginecologia e Obstetrícia, UFRGS. Gynecology Clinic of Hospital de Clínicas de Porto Alegre. M.D, PhD.

\section{AUTHORS' CONTRIBUTIONS}

JFLN, JLN, GOA, FNL, JPNL, VMFM, DPK, LFM: Conceptualization. JFLN, JNL, GSS, GOA, FNL, JPNL, VMFM, LFM, ADL, GPL, MEDC, TM, MPP: Data curation. JFLN, JNL, GSS, GOA: Formal Analysis. GSS, GOA, FNL, JPNL, VMFM, LFM, ADL, GPL, MEDC, TM, MPP: Investigation. JFLN, JLN, GSS, GOA, FNL, JPNL, VMFM, LFM, ADL, GPL: Methodology. JFLN, JNL: Project administration. JFLN, JLN, GSS, GOA, FNL, JPNL, VMFM, LFM, ADL, GPL, MEDC, TM, MPP, RR, AVP: Resources, Software. JFLN, JLN: Supervision. GSS, GOA: Validation. JFLN, JLN, GSS, GOA, FNL, JPNL, VMFM, DPK, LFM, ADL, GPL, MEDC, TM, MPP, RR, AVP: Visualization. JFLN, JNL, GSS, GOA, FNL, JPNL, VMFM, DPK, LFM, ADL, GPL, MEDC, MPP, TM, RR, AVP: Writing - original draft. JFLN: Writing - review \& editing. GSS, GOA: Both authors contributed equally to the development of the present study.

3. WHO Coronavirus Disease (COVID-19-19) Dashboard [cited in 2021 March 28] In: WHO int [Internet]. Available from: https:// COVID-1919.who.int.

4. Report of the WHO-China Joint Mission on Coronavirus Disease 2019 (COVID-19). In:WHO int [Internet]. 2020. [cited on March 21,2020].Available from: https://www.who.int/publications/i/item/ report-of-the-who-china-joint-mission-on-coronavirus-disease2019-(covid-19) 
5. Ministério da Educação (BR). Despacho de 29 de maio de 2020. DIÁRIO OFICIAL DA UNIÃO. 2020; June 1. Edition: 103. Section: 1. Page: 32.

6. Ministério da Educação (BR). Portaria n ${ }^{\circ} 544$, de 16 de junho de 2020. DIÁRIO OFICIAL DA UNIÃO. 2020; June 17. Edition: 114. Section: 1. Page: 62.

7. Wang D, Hu B, Hu C, Zhu F, LiuX, Zhang J, et al. Clinical characteristics of 138 hospitalized patients with 2019 novel coronavirus-infected pneumonia in Wuhan, China. JAMA. 2020;323(11):1061-69. https://doi.org/10.1001/jama.2020.1585

8. Ing EB, Xu QA, Salimi A, Torun N. Physician deaths from corona virus (COVID-19) disease. Occup Med. 2020;70(5):370-4. https:// doi.org/10.1093/occmed/kqaa088

9. Wong TW, Lee CK, Tam W, Lau JT, Yu TS, Lui SF et al. Cluster of SARS among medical students exposed to single patient, Hong Kong. Emerg Infect Dis. 2004;10(2):269-76. https://doi.org/10.3201/ eid1002.030452
10. Haque A, Mumtaz S, Khattak O, Mumtaz R, Ahmed A. Comparing the preventive behavior of medical students and physicians in the era of COVID-19: novel medical problems demand novel curricular interventions. Biochem Mol Biol Educ. 2020;48(5):473-81. https:// doi.org/10.1002/bmb.21406

11. Houlihan CF, VoraN,BirneT,Lewer D, Kelly G, Heaney J,etal. Pandemic peak SARS-CoV-2 infection and seroconversion rates in London frontline health-care workers. Lancet. 2020;396(10246):e6-7. https://doi.org/10.1016/S0140-6736(20)31484-7

12. Chou R, Dana T, Buckley DI, Selph S, Fu R, Totten AM. Epidemiology of and risk factors for coronavirus infection in health care workers: a living rapid review. Ann Intern Med. 2020;173(2):120-36. https:// doi.org/10.7326/M20-1632

13. Bampoe S, Lucas DN, Neall G, Sceales P, Aggarwal R, Caulfield $K$, et al. A cross-sectional study of immune seroconversion to SARS-CoV-2 infrontline maternity health professionals. Anaesthesia. 2020;75(12):1614-19. https://doi.org/10.1111/anae.15229 